\title{
An Improved Evolutionary Algorithm for Dynamic Vehicle Routing Problem with Time Windows
}

\author{
Jiang-qing Wang, Xiao-nian Tong, and Zi-mao Li \\ College of Computer Science, South-central University for Nationalities, \\ Wuhan, 430074, China
}

\begin{abstract}
The dynamic vehicle routing problem is one of the most challenging combinatorial optimization tasks. The interest in this problem is motivated by its practical relevance as well as by its considerable difficulty. We present an approach to search for best routes in dynamic network. We propose a dynamic route evaluation model for modeling the responses of vehicles to changing traffic information, a modified Dijkstra's double bucket algorithm for finding the real-time shortest paths, and an improved evolutionary algorithm for searching the best vehicle routes in dynamic network. The proposed approach has been evaluated by simulation experiment using DVRPSIM. It has been found that the proposed approach quite efficient in finding real-time best vehicle routes where the customer nodes and network information changes dynamically.
\end{abstract}

Keywords: Combinatorial Optimization, Dynamic Vehicle Routing Problem, Dijkstra Algorithm, Evolutionary Algorithm.

\section{Introduction}

The Vehicle Routing Problem (VRP) has been largely studied because of the interest in its applications in logistic and supply-chains management 122345. The VRP can be classified into two categories: static and dynamic 6]. The Dynamic Vehicle Routing Problem (DVRP) 78] is a richer problem compared to the static ones 9]. It not only involves increasing the problem size as new customer nodes enter the network but also changing in dynamic network, and highly sensitive to real-time traffic information. Most of current heuristic algorithms developed for the DVRP consider static traffic information, the travel time between customer nodes depend on distances only 1011 12 13. However, the best path from any given node to the final destination depends not only on the node but also on the arrival time at that node 14. Some of the researches have been concentrated on the development of real-time travel time functions [151617. In these approaches, traffic congestion and random fluctuation of traffic flow are not reflected.

The problem considered in this paper is the Dynamic Vehicle Routing Problem with Time Window (DVRPTW). We present an approach to search for best routes in dynamic network. The approach considers route attributes, real-time 
traffic information and dynamic demand information simultaneously, and can find the best vehicle routes for the DVRPTW.

The rest of the paper is organized as follows. Section 2 proposes the mathematical model of the DVRPTW. Section 3 develops a new route evaluation model. Using this model, a modified Dijkstra's double bucket algorithm is presented. Section 4 designs an improved evolutionary algorithm for the DVRPTW. Section 5 estimates the value of the developed approach. In Section 6 , we discuss conclusions.

\section{Mathematical Model of the DVRPTW}

The DVRPTW is given by a set of vehicles $K$, a special node called the depot, a set of customer nodes $V$, and a network connecting the depot and customers. For simplicity, we denote depot as customer 0 . Since each vehicle has a limited capacity $Q_{k}$, and each customer has a demand $q_{i}, Q_{k}$ must be greater than or equal to the summation of all demands on any route. Any customer $i$ must be serviced within a pre-defined time window $\left[T_{\text {start }_{i}}, T_{\text {end }_{i}}\right]$. Let us assume that $T_{i}$ is the arrival time of customer $i, T_{i, j}$ is the travel time between customer $i$ and $j, \alpha_{i}(i=1,2,3,4)$ is the penalty coefficient, $T$ is the end time period. We trade off the vehicle number, travel time, wait cost of vehicles and wait cost of customers in the objective function of the DVRPTW, which can be formulated as the following.

$$
\min \left(\alpha_{1} K+\sum_{k \in K}\left(\alpha_{2} \sum_{p=1}^{m_{k}} T_{i_{p-1}^{t}, i_{p}^{t}}+\alpha_{3} \sum_{p=0}^{m_{k}}\left(T_{\text {start }_{i_{p}^{t}}}-T_{i_{p}^{t}}\right)^{+}+\alpha_{4} \sum_{p=0}^{m_{k}}\left(T_{i_{p}^{t}}-T_{\text {end }_{i_{p}^{t}}}\right)^{+}\right)\right)
$$

Subject to

$$
\begin{gathered}
i_{m_{k}}^{t}=0, \forall k \in K \\
\sum_{j \in V} \sum_{t=0}^{T} x_{0 j}^{t}=\sum_{j \in V} \sum_{t=0}^{T} x_{j o}^{t}=K \\
\sum_{j \in V} \sum_{t=0}^{T} x_{i j}^{t}=\sum_{j \in V} \sum_{t=0}^{T} x_{j i}^{t}=1, i \in\{V-\{0\}\} \\
\sum_{p=0}^{m_{k}} q_{i_{p}} \leq Q_{k}, \forall k \in K
\end{gathered}
$$

Where:

$\left\{i_{0}^{t}, i_{1}^{t}, \ldots, i_{m_{k}}^{t}\right\}$ : the route of vehicle $\mathrm{k}$ at time $\mathrm{t}$, $x_{i j}^{t}=\left\{\begin{array}{l}1, \text { if any vehicle departures from customer } \mathrm{i} \text { to } \mathrm{j} \text { at time } \mathrm{t} \\ 0, \text { otherwise }\end{array}\right.$

$$
(x-y)^{+}=\max \{0, x-y\} .
$$




\section{Real-Time Shortest Path in Dynamic Network}

\subsection{Dynamic Route Evaluation Model}

A number of practical traffic information is selected, including real-time traffic information and route attributes, as multiple criteria for the developed dynamic route evaluation model.

1. Route length: the physical distance of the route.

2. Route width: the number of lanes in the route. The optimal speed of vehicles, Speed optimal, is based on lanes in the route when they travel along this route.

3. Route difficulty: turning movement is selected to measure the route difficulty.

4. Actual speed of vehicles on one route:

$$
\text { Speed }_{\text {actual }}=\alpha \text { Speed }_{\text {optimal }} \alpha \in[0,1]
$$

Where $\alpha$ is based on route difficulty, accident, traffic congestion, weather conditions, etc.

We use these multiple criteria to evaluate real-time travel times for each route in dynamic network, which can be categorized by several criteria. Firstly, it may be classified into two types: static and dynamic, according to how it is defined with respect to time. Secondly, it may be classified into two types: stochastic and deterministic, according to whether it is random variables or not. Lastly, it may also be classified into two value types: crisp and fuzzy (Table 1).

Table 1. Multiple Criteria for Route Evaluation

\begin{tabular}{lllll}
\hline Criteria & Unit & Certainty & Variability & Measurement \\
\hline Route length & $k m$ & DT & ST & Crisp \\
Route width & NM & DT & ST & Crisp \\
Route difficulty & NM & DT & ST & Crisp \\
Accidents & Level & SC & DN & Fuzzy \\
Traffic congestion & Level & SC & DN & Fuzzy \\
Weather conditions & Level & SC & DN & Fuzzy \\
\hline
\end{tabular}

NM: normalized, DT: deterministic, SC: stochastic, ST: static, DN: dynamic Real-time travel time on this route:

$$
\text { Travel time }=\text { Route length / Speed actual }
$$

\subsection{Real-Time Shortest Path Algorithm}

Here, we develop a modification of Dijkstra's double bucket algorithm for path finding in dynamic network. In the developed algorithm, the length of a path is defined to be the travel time on this path. In the problem we considered, a 
directed graph is given, $G=(V, E)$, where $V$ represents nodes and $E$ represents the route between two nodes. For each node $v \in V$, it is assigned a potential $d(v) \geq 0$, where in this case representing the current shortest time to the source node. For each edge $e \in E$, it is assigned a cost function $c(e) \geq 0$, representing current travel time between two nodes connected by the edge. The length of a path is now defined to be the summation of the cost of the edges on that path. There may be impedance on an edge corresponding to some traffic limitations, such as accidents, traffic congestion, and weather conditions. So $c(e)$ and $d(v)$ are time-varying according to real-time traffic information in a day. Other variables used by the algorithm are number of nodes, edges, low level buckets and largest normal travel time along a single edge which are denoted as n, m, B, C respectively.

The developed algorithm calculates the travel time between nodes when rerouting request is accepted. The output of the algorithm is the real-time shortest paths between nodes at that time. The time taken by the algorithm on a graph with $n$ vertices is $O(m+n(B+C / B))$, and by the standard Dijkstra's algorithm, it is $O\left(n^{2}\right)$. This characteristic is very important for the DVRP.

\section{Improved Evolutionary Algorithm (IEA) for the DVRPTW}

\subsection{Representation}

We use the modified form of random keys representation [18. In our representation, a chromosome consists of genes and each gene represents a customer node. The customer nodes have fixed gene positions in the chromosomes and the order in which they are visited is determined by sorting on the gene values. The random keys have information about the vehicle number used for a service and the value for sorting, where the digit before the point represents the vehicle number and the digit after the point are used as sort keys to decode visiting sequence. For example, a chromosome to 8 customers problem may be:

$\begin{array}{llllllll}1 & 2 & 3 & 4 & 5 & 6 & 7 & 8\end{array}$

1.3242 .3152 .7612 .1891 .4361 .8471 .8751 .104

The route for the previous chromosome can be represented as follows.

Vehicle $_{1}: 8 \rightarrow 1 \rightarrow 5 \rightarrow 6 \rightarrow 7$, Vehicle $2: 4 \rightarrow 2 \rightarrow 3$

The steps for generating chromosome are as follows.

1. Generate the vehicle number.

2. Generate sorting number.

3. Combine the vehicle number and sorting number.

\subsection{Handling of the Constraints}

The initial population of chromosomes is generated randomly and may not satisfy the constraints of the proposed DVRPTW. And some new chromosomes generated after genetic operators (crossover, mutation) may not satisfy the constraints. So, the constraints-checking steps are executed after new chromosomes 
being generated. We consider soft time windows in this paper, so we use the penalty method. The infeasible chromosomes have fewer opportunities than the feasible chromosomes, and have the chances to be turned to the feasible chromosomes by the genetic operator.

\subsection{Crossover Operator}

We use the two-points crossover operator. It is assumed that there are two chromosomes $p_{1}, p_{2}$ as follows and two generated crossover points are 2 and 5 .

$p_{1}: 1.3242 .3152 .7612 .1891 .4361 .8471 .8751 .104$

$p_{2}: 2.1341 .5162 .3852 .0341 .8911 .6252 .3291 .618$

After the crossover operation, two children $c_{1}$ and $c_{2}$ are generated as follows.

$c_{1}: 1.3242 .315 \mathbf{2 . 3 8 5} \mathbf{2 . 0 3 4} \mathbf{1 . 8 9 1} 1.8471 .8751 .104$

$\mathrm{c}_{2}: 2.1341 .516 \mathbf{2 . 7 6 1} \mathbf{2 . 1 8 9} \mathbf{1 . 4 3 6} 1.6252 .3291 .618$

\subsection{Mutation Operator}

The mutation operator changes the vehicle number with a newly generated vehicle number and does not change the information that is used as sorting key, because the sequence of the customer nodes can be changed by just changing the assigned vehicle number of a customer. Let us assume that there are one chromosomes $p_{1}$ as follows and the mutation point is 6 .

$p_{1}: 1.3242 .3152 .7612 .1891 .4361 .8471 .8751 .104$

After the mutation operation, the children $\mathrm{c}_{3}$ is generated as follows.

$\mathrm{c}_{3}: 1.3242 .3152 .7612 .1891 .4362 .8471 .8751 .104$

\section{Experimental Results and Analysis}

In the literature, there is no commonly used benchmark for the DVRPTW, so the authors have generated their own Dynamic Vehicle Routing Problem SIMulator(DVRPSIM) to evaluate the benefits of the developed approach. The simulated system of the DVRPTW is made up of three modules, route evaluation module, shortest path module and routing plan module, as shown in Fig.1.

The main function of route evaluation module is to evaluate the actual travel times of vehicles on each route using the real-time traffic information and the route attributes, to transmit the result to the shortest path module to determine the real-time shortest path between customers. According to the result obtained from the shortest path module, routing plan module based on IEA can determine the best routes for vehicles whenever requested. Vehicles will use these routes and drive on them in the dynamic network. When the rerouting requests are accepted, the system will determines the real-time best routes for vehicles.

In order to compare IEA with other algorithms, we choose two famous algorithms, Branch-Bound algorithm and Clarke-Wright algorithm, as benchmarks. We listed the comparison results in Table 2. 


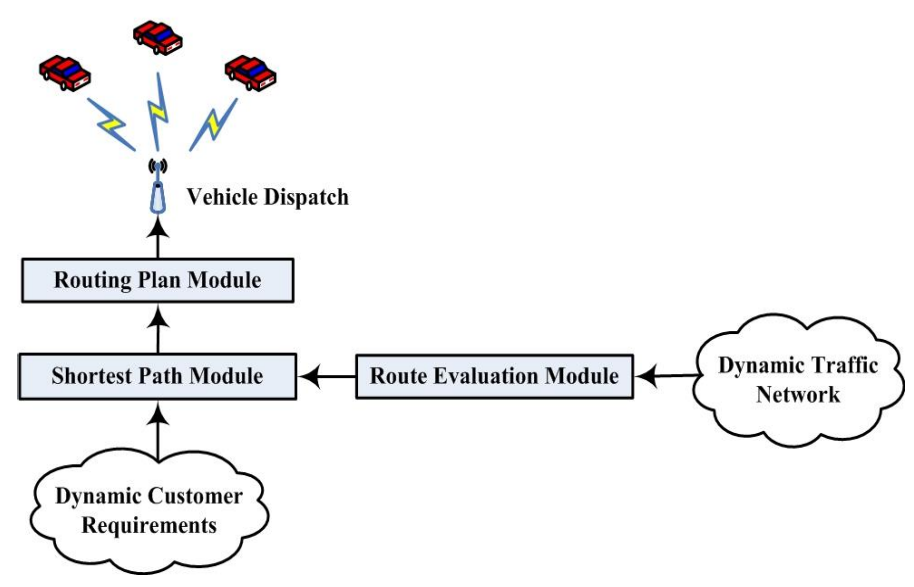

Fig. 1. Architecture of the Simulated System

Table 2. Results of Different Algorithms

\begin{tabular}{llll}
\hline & IEA & B-B & C-W \\
\hline Number of vehicle & 4 & 4 & 4 \\
Route Cost & 2808 & 2737 & 2907 \\
Wait Cost of Vehicles & 49 & 32 & 58 \\
Wait Cost of Customers & 27 & 33 & 53 \\
Total Cost & 5924 & 5749 & 6227 \\
Calculation Time & 63.117 & 512.096 & 50.673 \\
\hline
\end{tabular}

Table 2 shows that, compared with B-B, the calculation time of IEA is much lower and the route cost is a little bigger. And compared with $\mathrm{C}-\mathrm{W}$, its route cost is better and the calculation time is similar. Fig. 2 shows the cost comparison of these algorithms, and Fig. 3 shows the time comparison of them.

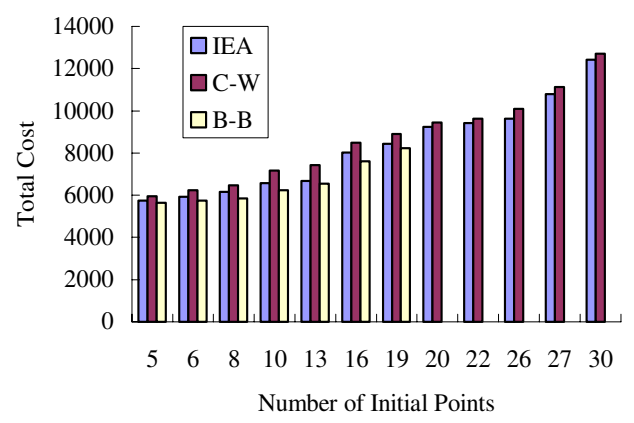

Fig. 2. Cost Comparison of Three Algorithms 


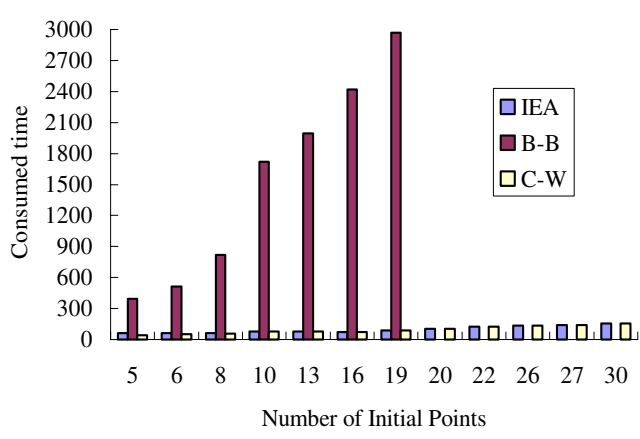

Fig. 3. Time Comparison of Three Algorithms

\section{Conclusions}

This paper presents an approach for the DVRPTW. We have proposed a dynamic route evaluation model to evaluate routes using route attributes and real-time traffic information. We have developed a modified Dijkstra's algorithm for finding real-time shortest paths in dynamic network. We have designed an improved evolutionary algorithm for searching the best vehicle routes of the DVRPTW. We have performed a simulation test using DVRPSIM. In the simulation test, we have compared three algorithms: IEA, B-B, and C-W. Our primary conclusion is that the developed approach based on IEA can find the best vehicle routes for the DVRPTW efficiently.

Acknowledgement. The authors gratefully acknowledge the financial support of the National Natural Science Foundation of China under Grant No.60603008.

\section{References}

1. Tighe, A., Smith, F.S., Lyons, G.: Priority based solver for a real-time dynamic vehicle routing. In: Systems, Man and Cybernetics, 2004 IEEE International Conference on. Volume 7. (2004) 6237-6242

2. Donati, A.V., Montemanni, R., Gambardella, L.M., Rizzoli, A.E.: Integration of a robust shortest path algorithm with a time dependent vehicle routing model and applications. In: Computational Intelligence for Measurement Systems and Applications, 2003. CIMSA '03. 2003 IEEE International Symposium on. (2003) 26-31

3. Tan, K.C., Lee, T.H., Chew, Y.H., Lee, L.H.: A multiobjective evolutionary algorithm for solving vehicle routing problem with time windows. In: Systems, Man and Cybernetics, 2003. IEEE International Conference on. Volume 1. (2003) 361-366

4. Tan, K.C., Lee, T.H., Ou, K., Lee, L.H.: A messy genetic algorithm for the vehicle routing problem with time window constraints. In: Evolutionary Computation, 2001. Proceedings of the 2001 Congress on. Volume 1. (2001) 679-686 vol. 1 
5. Alvarenga, G.B., Mateus, G.R.: A two-phase genetic and set partitioning approach for the vehicle routing problem with time windows. In: Hybrid Intelligent Systems, 2004. HIS '04. Fourth International Conference on. (2004) 428-433

6. Psaraftis, H.N.: Dynamic vehicle routing: Status and prospects. Annals of Operations Research 61(1995) (1995) 143-164

7. Alvarenga, G.B., de Abreu Silva, R.M., Mateus, G.R.: A hybrid approach for the dynamic vehicle routing problem with time windows. In: 5th International Conference on Hybrid Intelligent Systems (HIS 2005). (2005) 61-67

8. Song, J., Hu, J., Tian, Y., Xu, Y.: Re-optimization in dynamic vehicle routing problem based on wasp-like agent strategy. In: Intelligent Transportation Systems, 2005. Proceedings. 2005 IEEE. (2005) 231-236

9. Qiang, L.: Integration of dynamic vehicle routing and microscopic traffic simulation. In: Intelligent Transportation Systems, 2004. Proceedings. The 7th International IEEE Conference on. (2004) 1023-1027

10. Lou, S.Z., Shi, Z.K.: An effective tabu search algorithm for large- scale and realtime vehicle dispatching problems. In: Machine Learning and Cybernetics, 2005. Proceedings of 2005 International Conference on. Volume 6. (2005) 3579-3584

11. Del Bimbo, A., Pernici, F.: Distant targets identification as an on-line dynamic vehicle routing problem using an active-zooming camera. In: Visual Surveillance and Performance Evaluation of Tracking and Surveillance, 2005. 2nd Joint IEEE International Workshop on. (2005) 97-104

12. Tian, Y., Song, J., Yao, D., Hu, J.: Dynamic vehicle routing problem using hybrid ant system. In: Intelligent Transportation Systems, 2003. Proceedings. 2003 IEEE. Volume 2. (2003) 970-974

13. Ce, F., Hui, W., Ying, Z.: Solving the vehicle routing problem with stochastic demands and customers. In: Parallel and Distributed Computing, Applications and Technologies, 2005. PDCAT 2005. Sixth International Conference on. (2005) $736-739$

14. Seongmoon, K., Lewis, M.E., White, C. C., I.: Optimal vehicle routing with realtime traffic information. Intelligent Transportation Systems, IEEE Transactions on 6(2) (2005) 178-188

15. Jung, S.J.: A Genetic Algorithm for the Vehicle Routing Problem with Time Dependent Travel Times. PhD thesis, University of Maryland, USA (2000)

16. Fischetti, M., Laporte, G., Mattello, S.: The delivery man problem and cumulative matroids. Operation Research 41 (1993) 1055-1076

17. Malandraki, C., Daskin, M.S.: Time dependent vehicle routing problems: Formulations, properties and heuristic algorithms. Transportation Science 26(3) (1992) $185-200$

18. Bean, J.: Genetic algorithms and random keys for sequencing and optimization. ORSA Journal on Computing 6(2) (1994) 154-160 